\title{
¿Cómo usar diccionarios bilingües en línea? Ejemplos para el desarrollo de las competencias lexicográficas en la enseñanza del alemán como lengua extranjera ${ }^{1}$
}

\author{
María Egido Vicente \\ Universidad de Salamanca \\ mariaegido@usal.es
}

Meike Meliss

Universidad de Santiago de Compostela

meike.meliss@usc.es

Resumen: Este artículo expone a partir de una serie de ejemplos diferentes situaciones de uso del diccionario bilingüe que evidencian la importancia de llevar a cabo una adecuada adquisición y desarrollo de las competencias lexicográficas en el contexto de enseñanza-aprendizaje de lenguas extranjeras y, en este caso en concreto, del alemán como lengua extranjera. Con este propósito se parte de tres competencias básicas: la selección de la obra lexicográfica adecuada según la situación comunicativa, la desambiguación pertinente en el contexto de la recepción en L2 y traducción de L2 a L1 y la selección y uso del equivalente en el contexto de la producción y traducción en la L2. El objetivo de esta aportación es poner de manifiesto la necesidad de identificar adecuadamente por parte del usuario de un recurso lexicográfico bilingüe la información lexicológica pertinente a la forma, contenido y uso de los lemas consultados tanto en la situación de recepción y producción en L2 como en el contexto de la traducción de y a L2.

1. Este trabajo se ha elaborado en el marco del proyecto de investigación COMBIDIGILEX (FFI201564476-P y FEDER) y en relación con la red de investigación lexicográfica RELEX ED431DR 2016/06 (Xunta de Galicia). 
Para ello, haciendo hincapié en el valor del diccionario como obra de consulta y aprendizaje, es fundamental no solo identificar la información explícita en el artículo lexicográfico, sino también la implícita, especialmente en el caso de los diccionarios bilingües en formato online.

Palabras clave: lexicografía bilingüe online, competencias lexicográficas, didáctica lexicográfica, enseñanza del alemán como lengua extranjera.

Abstract: This paper explains on the basis of a set of examples different scenarios that point out the importance of the correct acquisition and development of the lexicographical competences in the teaching and learning context of a foreign language, in this case, German as a foreign language. For this purpose, three basic competences are selected: the selection of the appropriate lexicographic work according to the communicative situation, the search and selection of the equivalent in the context of $\mathrm{L} 2$ reception and $\mathrm{L} 2$ translation and the selection and use of the equivalent in L2 in the context of production and translation in L2. The aim will be to highlight the need for a clearer identification of the lexicographical information relating to the form, meaning and use of the searched word both in the reception and production situation in L2 and in the context of the translation from and to L2. For this purpose, it is essential to emphasize the role of the dictionary as a consultation and learning tool, not only to identify the explicit information in the lexicographical article.

Keywordsः online bilingual lexicography, lexicographical competences, lexicography didactics, teaching German as a foreign language.

Recibido el 12/06/2017

Aceptado el 20/10/2017 


\section{Introducción}

A pesar del cada vez más alto grado de aceptación de herramientas en torno al tratamiento (semi)automático de las lenguas como son, por ejemplo, los servicios de traducción automática o las bases de datos terminológicos con acceso en línea, el uso del diccionario y, en concreto, del diccionario bilingüe (=DB) sigue siendo una práctica extendida entre los aprendices de lenguas extranjeras. El principal objetivo de la consulta es el deseo de resolver dudas en situaciones de codificación y decodificación, así como en el proceso de traducción directa e inversa. En el caso de la enseñanza del alemán como lengua extranjera $(=\mathrm{DaF})$, estudios recientes en el campo de la lexicografía y la didáctica lexicográfica, como por ejemplo la encuesta DICONALE-DIC ${ }^{2}$, confirman esta tendencia. En este contexto, la frecuencia de uso del DB decir supera de forma generalizada la de los diccionarios de aprendizaje monolingües y diccionarios específicos (Domínguez et alii 2013: 146, Fernández et alii 2016, Flinz 2014, Meliss 2015b).

La constante discusión teórica en torno a los DB llevada a cabo desde los años noventa y, en particular, en torno al par de lenguas alemán-español (Hausmann 1991, Hausmann y Werner 1991, Fuentes 1997, Meyer y Wiegand 2000, Model 2010, Meliss 2013) ha contribuido a su paulatina mejora (Fuentes 2015: 295). En la actualidad contamos además con una serie de nuevos recursos multilingües en línea que ofrecen diversas combinaciones de lenguas y que comparten, entre otras, la característica de no estar basados en una versión print precedente (p.ej.: Leo, Bab.la, dict.cc, Linguee, Linguatools).

En el contexto general de aprendizaje de segundas y terceras lenguas, enmarcado en la educación secundaria y superior, la consulta lexicográfica en formato print ocupa en la actualidad una posición periférica, sustituida casi en su totalidad por la consulta en diccionarios online (Fernández et alii 2016: 79-80, Meliss 2015b: 406). Junto a factores como la rapidez, inmediatez o el acceso gratuito, el diccionario bilingüe online (=DBO) destaca sobre todo por su carácter multimedial, hipertextual y participativo-colaborativo ${ }^{3}$. La multimedialidad permite al usuario obtener un input auditivo y hasta audiovisual que, sin duda, favorece la asimilación y buen uso de la información lexicográfica. En cuanto a la hipertextualidad, esta cualidad intrínseca al medio online permite al usuario ampliar el

2. Encuesta sobre los hábitos de los usuarios de diccionarios en el ámbito del alemán y del español como lenguas extranjeras elaborada en el marco del proyecto de investigación DICONALE-online (MINECO \& FEDER: FFI2012-32658). Los resultados se publicaron en Meliss (2015b) y en Fernández et alii (2016).

3. Cabe destacar que el incipiente interés por el medio hipertextual en el ámbito lexicográfico (Engelberg y Lemnitzer ${ }^{4} 2009: 220$, Schmitz 2013: 1013) se ve reforzado por una floreciente investigación en el campo de la lexicografía online (cfr. Fuertes-Olivera y Bergenholtz 2011, Grouws et alii 2013, Klosa 2008, Klosa y MüllerSpitzer 2016, Mann 2014, Storrer 2010, Tarp 2013). 
espectro de la búsqueda mediante hipervínculos que dan acceso a numerosos recursos online, como por ejemplo otros diccionarios de diversa tipología (monolingües, especializados, paradigmáticos, sintagmáticos, etc . $_{+}$, enciclopedias, glosarios o corpus lingüísticos ${ }^{4}$. El formato online permite además una consulta interactiva por medio de la participación en foros o espacios colaborativos ${ }^{5}$.

En el contexto específico de la enseñanza de DaF, el uso de recursos lexicográficos bilingües o multilingües en formato online supera el de los recursos tradicionales ${ }^{6}$, a la vez que se identifica una mayor frecuencia de uso de las correspondientes aplicaciones web para la telefonía móvil (Fernández Méndez et al. 2016, Meliss 2015b, Nied-Curcio 2014).

El nuevo contexto que proporciona la lexicografía bilingüe online no es, sin embargo, garantía de una consulta satisfactoria. Junto con las competencias lingüísticas pertinentes y al igual que sucede con los diccionarios en formato print, el usuario necesita de las competencias lexicográficas clásicas, así como de otras adaptadas al nuevo formato que eviten su perdida y desorientación en el medio hipertextual (Hass y Schmitz 2010٪4). Por esta razón, junto con el desarrollo de diccionarios cada vez más intuitivos y manejables, la inclusión de una didáctica de la lexicografía general y lexicografía online en particular y la consecuente adquisición de las competencias lexicográficas se perfila como una conditio sine qua non para el desarrollo satisfactorio del proceso de consulta en todas las situaciones posibles.

El objetivo del presente artículo es seleccionar y exponer diferentes situaciones que deberían ser tenidas en cuenta para el desarrollo de las competencias lexicográficas de un usuario hispanohablante (L1 = español) que estudia alemán como lengua extranjera $(\mathrm{L} 2=\mathrm{DaF})$. Con este fin, partiendo de ejemplos concretos, diferenciamos tres competencias básicas: (i) la selección del DBO adecuado según la situación comunicativa, (ii) la consulta y desambiguación en el contexto de la recepción en L2 y (iii) la selección y uso del equivalente en el contexto de la producción y traducción en la L2 (alemán). En este trabajo nos centramos únicamente en los DB con acceso online, ya que, según lo arriba indicado, se trata del formato preferido por los usuarios hispanohablantes en ámbitos de DaF. Para ello nos basamos en dos diccionarios bilingües de aprendizaje para el par de

4. Cfr. Heid (2013) y Abel y Lemnitzer (2014).

5. Cfr. Meyer y Gurevych (2014) y Melchior (2012).

6. Consúltense, entre otros, los datos obtenidos en la encuesta UPDALE (Domínguez et alii 2013) y DICONALE-DIC (Fernández et alii 2016, Meliss 2015b) para el par de lenguas alemán-español. Por otro lado, encuestas sobre hábitos lexicográficos realizadas para el par de lenguas alemán-inglés indican que alrededor del $40 \%$ de los consultados siguen utilizando diccionarios en formato print en casos concretos (Koplenig y Müller-Spitzer 2014: 140). Un resultado semejante expone Flinz (2014: 218) en el contexto de la enseñanza $\mathrm{DaF}$ a italohablantes. 
lenguas alemán-español de grandes editoriales con antecedentes print y de acceso online gratuito como son Pons-online (P-DBO) y Langenscheidt-online (L-DBO), así como en un diccionario online, también de acceso gratuito, que no cuenta con un antecedente en formato print, pero que también goza de amplia aceptación entre los aprendices: Leo.

\section{El desarrollo de las competencias lexicográficas en el ámbito de uso de los DBO}

Con el fin de sacarle el mayor rendimiento posible al DB y, en especial, al DBO, es necesario que el usuario desarrolle una competencia lexicográfica específica según el formato de la obra lexicográfica seleccionada y sus intereses comunicativos. Esta necesidad se ve multiplicada en el caso de los DBO, ya que el formato en línea permite diferentes formas de acceso y consulta, además de múltiples estructuras e interrelaciones complejas de los datos lingüísticos. Asimismo, la obra lexicográfica bilingüe no solamente se debe entender como una simple obra de consulta, sino también como una herramienta o recurso de aprendizaje bajo la premisa de la adecuada decodificación de la información explícita e implícita por parte del usuario (Egido 2011: 46-49, Fernández 2015: 355). Desde un enfoque contrastivo, el DB debería ayudar al aprendiz a tomar conciencia sobre las posibles convergencias, divergencias y asimetrías entre la lengua de partida y la lengua meta y, de esta forma, favorecer la asimilación del funcionamiento del nuevo sistema lingüístico partiendo del que ya conoce (Fuentes 2015: 285).

De acuerdo con su naturaleza hipertextual y la cantidad y calidad de la información que ofrecen los recursos en línea a nivel micro- y macroestructural, los diccionarios aportan datos explícitos e implícitos relevantes para las diferentes situaciones de uso, tales como son la codificación y decodificación de un texto o la traducción directa e inversa según el contexto comunicativo de la consulta. La didáctica lexicográfica tiene la función de desarrollar la competencia lexicográfica del usuario con el fin de facilitar su acceso al conjunto de información recogida en el diccionario, ya sea en relación a contenidos léxicos, gramaticales o socioculturales.

A continuación se describen y ejemplifican brevemente las tres competencias lexicográficas básicas, cuyo dominio, a nuestro juicio, debería garantizar un proceso de consulta satisfactorio en el ámbito de uso de los DBO. 


\section{Competencia 1: selección del DBO adecuado según la situación comunicativa}

Desde nuestra experiencia como docentes de lenguas extranjeras observamos que muchos de los errores de consulta derivados de un mal uso del DB tienen su origen en una fase previa a la propia consulta del diccionario, concretamente, en una mala selección de la obra lexicográfica. En el caso de los DBO hay que considerar especialmente algunos aspectos relacionados con su carácter hipertextual. $\mathrm{A}$ continuación proponemos algunos de los criterios que se pueden tener en cuenta a la hora de seleccionar un DBO. Para ello partimos de los estudios de Kemmer (2010) y de la propuesta de Meliss (2016b) con el fin de diferenciar entre ocho dimensiones (D1-D8) que intentan dar respuesta a las siguientes preguntas:

D1: ¿Podemos fiarnos de la información lexicográfica que aporta el diccionario?

D2: ¿Quién usa el diccionario y para qué?

D3: ¿Cómo se presenta la información?

D4: ¿Cómo se accede a la información? ${ }^{7}$

D5: ¿Cómo está estructurada la información?

D6: ¿Qué recursos multimedia se ofrecen?

D7: ¿Qué posibilidades de interacción y participación colaborativa existen?

D8: ¿Qué vínculos internos y externos existen?

\footnotetext{
7. El usuario debe saber que en alemán existen ciertas ambigüedades a nivel ortográfico. Sobre todo en determinados extranjerismos se admite una doble realización ortográfica, aunque, generalmente, existe una preferencia por una u otra forma (Yoghurt/Yogurt, Ketschup/Ketchup, etc.). A la hora de decodificar un texto en alemán, el usuario puede encontrar no solamente alguna palabra realizada ortográficamente en una forma poco frecuente o inusual, sino incluso incorrecta según las reglas ortográficas vigentes (p.ej.: *Schlo $\beta \rightarrow$ castillo/ cerradura). Los diccionarios online están perfilando mecanismos que permiten encontrar palabras con un margen de error ortográfico, como es el caso en el ejemplo indicado, cuya ortografía actual obliga la substitución de $<\beta>$ por $<$ ss $>$. 8. Cfr. Engelberg y Lemnitzer ( ${ }^{4} 2009: 74$ ss.) y Engelberg y Müller-Spitzer (2013).
} 


\section{Competencia 2 : consulta y desambiguación en el contexto de la recepción en L2}

En el caso de la recepción de un texto escrito u oral en L2 y la necesidad de una consulta lexicográfica para una correcta decodificación del mensaje en L2 el usuario requiere, según el grado de contextualización, la adecuada desambiguación en todos los niveles lingüísticos; es decir, a nivel formal, a nivel del contenido y a nivel pragmático. Este proceso es necesario para entender el significado contextualizado y disponer de la información que permita establecer una relación de equivalencia entre el lexema de la lengua de partida (alemán: L2) y la lengua de llegada (español: L1). A continuación se exponen seis situaciones en las que no solo se refleja una selección de problemas a los que se enfrenta el usuario de un DB a la hora de decodificar un texto en alemán como L2 (en ocasiones para su posterior traducción); sino que también se pone de manifiesto la posibilidad de usar el recurso lexicográfico como herramienta de aprendizaje de la lengua extranjera.

Situación 1: en la recepción de un texto escrito puede surgir la necesidad de diferenciar entre homógrafos (p.ej.: <modern > (adjetivo: moderno/ verbo: pudrirse), $\left\langle\right.$ rasten $>$ (verbo $3^{\text {a }}$ pers. pl. imp. del verbo rasen $=$ correr $/$ verbo $3^{\mathrm{a}}$ pers. $\mathrm{pl}$. pres. indic. del verbo rasten $=$ pausar $/$ descansar). El usuario debe entender que ambos lexemas poseen diferentes significados, además de presentar divergencias en otros ámbitos, como por ejemplo en el fónico o, en el caso de $\langle$ modern $\rangle$, en el morfosintáctico al tratarse de diferentes clases de palabras. Para percatarse de ello, el usuario de P-DBO no solo tiene que tener conocimientos del IPA, sino también conocer los signos diacríticos para entender que ambos lemas tienen diferente acentuación. También tendría que poseer una buena comprensión auditiva para percibir las diferencias mediante el archivo de audio, así como de conocimientos básicos de las características morfosintácticas de cada clase de palabra. En el caso de <rasten> llama la atención que en P-DBO, L-DBO y Leo al teclear la forma verbal el usuario no solamente encuentre información para el verbo rasten (descansar, posar), sino también para rasen (correr) (cfr. entrada 1). Los conocimientos sobre la flexión regular de los verbos alemanes y el contexto ayudan al usuario a seleccionar el lema verbal adecuado. En caso de duda los links externos a las tablas de conjugación que ofrecen los diccionarios P-DBO y Leo pueden ser de gran utilidad. 
Entrada 1: artículo lexicográfico de rasten en L-DBO (fragmento)

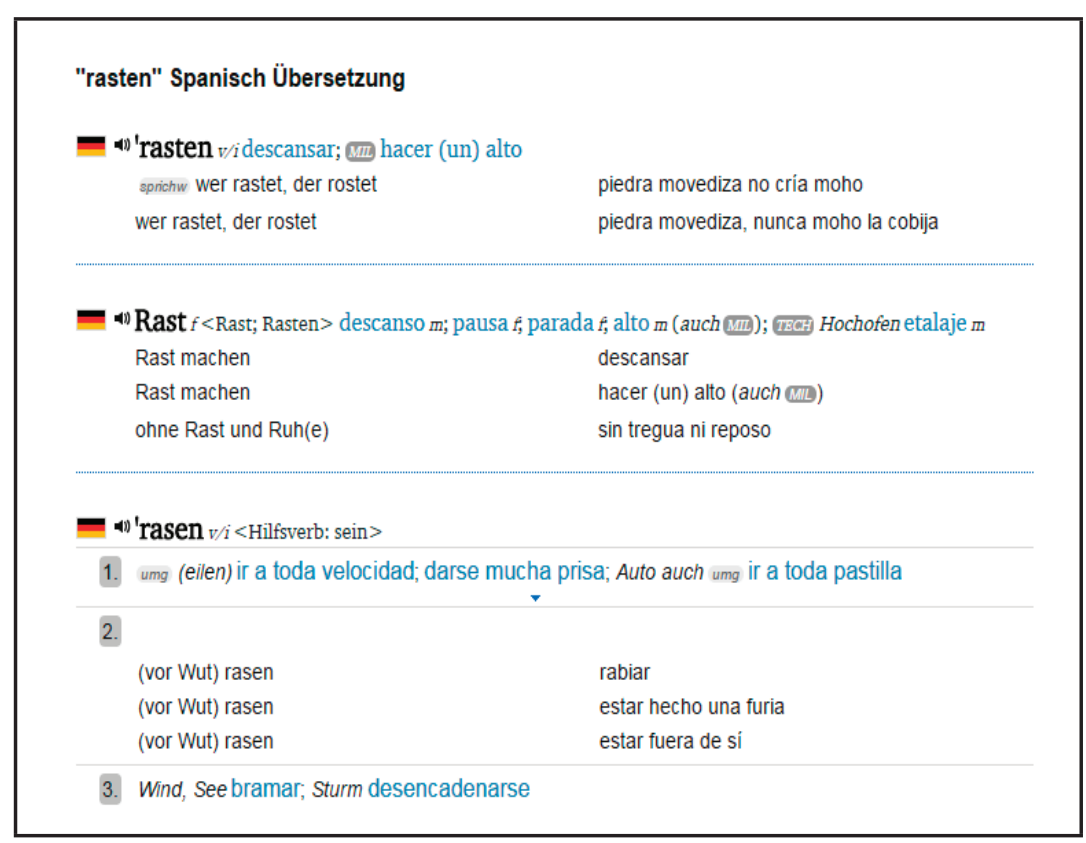

Situación 2: algunos verbos prefijados del alemán poseen una variante separable y otra inseparable con sus respectivos cambios semánticos, en ocasiones, también morfológicos (<übersetzen>, <umfahren>, etc.). Cuando el usuario tiene que decodificar el significado contextualizado de übersetzen (traducir/ cruzar) encuentra al menos dos entradas diferentes con sus respectivos equivalentes. También recibe información sobre las divergencias sintácticas de ambas acepciones mediante la marca de (in)transitividad e incluso sobre el uso del auxiliar para las formas verbales compuestas. La información lexicográfica y la contextualización suelen ser suficientes para que el usuario pueda desambiguar ambas acepciones y encontrar el equivalente correspondiente. $\mathrm{Si}$, por el contrario, se interesa más a fondo por el carácter separable del prefijo "über-" debe disponer de unos conocimientos gramaticales determinados que le permitan interpretar correctamente la información lexicográfica que se presenta de forma implícita en los diccionarios consultados. En P-DBO se marca el carácter separable del prefijo mediante el símbolo "|", cuyo significado debe ser interpretado adecuadamente por el usuario para poder diferenciar ambos lemas verbales, ya que ningún ejemplo de uso indica esta característica a excepción del siguiente: de. Sie hat das Buch vom Deutschen ins Englische übersetzt (es. [Ella] ha traducido el libro del alemán al inglés), que aporta información implícita sobre el carácter inseparable mediante la forma del 
participio de esta variante. Por otra parte, este diccionario ni transcribe ni indica la discrepancia en la acentuación entre ambos lemas. Se apela, pues, al conocimiento gramatical del usuario, que, generalmente, aprende que los verbos con un prefijo separable llevan el acento en el propio prefijo. En el caso de L-DBO se renuncia, sin embargo, a la simbolización del carácter separable del prefijo „über" en übersetzen (cruzar, transbordar). Aquí se remite también al conocimiento gramatical del usuario, ya que marca la acentuación diferente y vincula el carácter del prefijo inseparable con la información sobre la forma del participio de pretérito que no lleva el morfema "ge“ (cfr. entrada 2 ).

Entrada 2: artículo lexicográfico de übersetzen en L-DBO (fragmento)

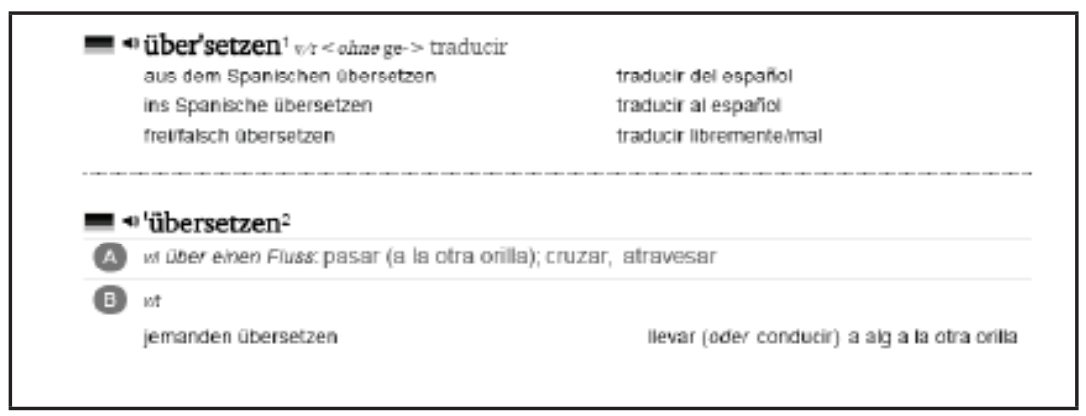

Situación 3: para un correcto proceso de desambiguación, el usuario debe saber además que sustantivos homonímicos y polisémicos se diferencian a menudo por la marca de género (das/ der Tor, der/ das Band, etc.) y un diferente paradigma de flexión que se visualiza mediante la forma del genitivo singular $y$ el nominativo plural. Tanto P-DBO como L-DBO ofrecen esta información y garantizan, junto con la información contextualizada, poder desambiguar y decodificar el significado de una u otra forma. La estructuración de la información en Leo sigue otras pautas, ya que no agrupa las diferentes acepciones de manera que únicamente un usuario observador sería capaz de percatarse de las diferencias de género y número entre das Tor (género neutro), en español puerta, portería o portal y der Tor (género masculino), en español necio (cfr. entrada 3). 
Entrada 3: artículo lexicográfico de Tor en Leo (fragmento)

\begin{tabular}{|c|c|c|}
\hline \multicolumn{3}{|c|}{ Substantive } \\
\hline (C) el pontón & Z1() das Tor Pl: die Tore & - Del endot Hove oft. \\
\hline (־) el gal [dep.] & प1(1) das Tor Pl: di Tore & Fussor, Handsay \\
\hline (1) la parterfia [dep.] & Z1(1) das Tor Pl: dis Tore & \\
\hline (1) el necio I la necia & T1(1) der Tor F: dis Torn & \\
\hline (-) is puerta tambien: [elec Intorm] & Z1) das Tor fl: die Tore & \\
\hline (1) el portalion & Z1) das Tor Pl: die Tore & \\
\hline (1) la puerta [dep] & Z1(1) das Tor P1: di Tore & 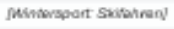 \\
\hline (1) ا mubipuerta [elec.] & (1) das n-Tor & \\
\hline
\end{tabular}

Situación 4: las diferentes acepciones de un verbo suelen ser desambiguadas mediante criterios sintácticos. Tanto P-DBO como L-DBO agrupan las acepciones verbales según la (in)transitividad del verbo o su carácter impersonal o reflexivo. En un proceso de decodificación el usuario sabe, generalmente, que la estructuración de los datos lexicológicos en los diccionarios se desarrolla en base a este criterio. Leo sigue, sin embargo, una estructuración diferente. En el lado alemán indica diferentes esquemas oracionales cuya formalización requiere conocimientos sintácticos previos de la gramática del alemán. Solo entonces el usuario puede relacionar el esquema jmdm etw. ${ }^{\text {akk }}$ geben, que indica que el verbo alemán requiere dos actantes, uno con el rasgo [+hum] (referido a "humano") en dativo y otro con el rasgo [+mat] (referido a «material») en acusativo, con el esquema argumental del verbo correspondiente en español: dar algo a alguien y enlazarlo con su conocimiento como hablante nativo del español acerca de las características morfosintácticas de ambos complementos (directo e indirecto) (cfr. entrada 4).

\section{Entrada 4: artículo lexicográfico de geben en Leo (fragmento)}

\begin{tabular}{|c|c|}
\hline \multicolumn{2}{|c|}{ Vorsen } \\
\hline (1) dar sipo a aiguien & 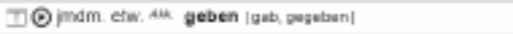 \\
\hline (1) haber - soo mparsanur rey - exisar & 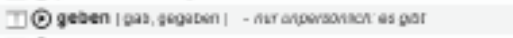 \\
\hline (1) impartr algo & 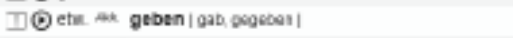 \\
\hline (1) largar algo a alguien - towitate, poipe at: & 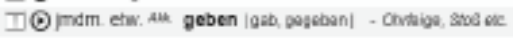 \\
\hline
\end{tabular}

Situación 5: la información sobre el estilo, registro y contextualización del lema ayuda a su correcta recepción y adecuada traducción. Para ello, el usuario tiene que conocer las diferentes marcas diastráticas que usa el diccionario. Los lexemas verbales gucken (mirar) o futtern (zampar) llevan la marca "umg./ fam." (informal/ familiar) en los diccionarios consultados. Lexemas como kotzen (vomitar/ potar) llevan la marca "vulgär/ derb/ salopp" (vulgar), mientras que otros como Antlitz (rostro) pertenecen a un registro elevado o culto (geh.). El usuario también debe entender las informaciones sobre los ámbitos de uso. Así una de 
las acepciones de Tor (portería/ gol) pertenece al ámbito del deporte (cfr. entrada 3) y una de las acepciones de Knete/ Koble (dinero/ pasta) al ámbito del lenguaje juvenil. También es necesario interpretar adecuadamente las marcas connotativas como por ejemplo "abw." (peyorativo) en el verbo labern (hablar mucho y sin sentido). ${ }^{9}$

Situación 6: en contextos orales el usuario debe establecer una relación entre lo que escucha y su correspondiente representación grafemática para poder realizar una consulta lexicográfica adecuada. En alemán existen ciertas peculiaridades entre la relación fonema-grafema. Para el desarrollo de una mejor competencia lexicográfica es, sin duda, de gran importancia incidir en fenómenos como los homófonos (['lerça] die Lerche/ Lärche), la Auslautverhärtung ([korp] der Korb), la realización del Schwa-Laut (['zonə] die Sonne), la existencia de una "R" vocálica

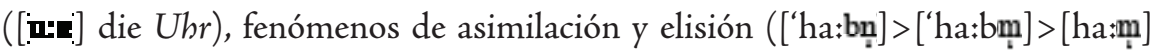
haben) y ciertas variaciones vinculadas a la realización fónica de extranjerismos ([bal'k可, bal'ko:n, bal'kõः] der Balkon). Sin estos conocimientos gramaticales, puede ser difícil para el usuario establecer una relación entre lo que percibe oralmente y la representación grafemática que le permite realizar una consulta lexicográfica.

\section{Competencia 3: selección y uso del equivalente en el contexto de la producción y traducción en la L2 (alemán)}

A pesar de que el DB es una herramienta bien conocida por los aprendices de lengua extranjera, los errores en el proceso de producción y traducción en la L2, derivados de un mal uso del DB, son bastante frecuentes. Nos referimos a frases como *ich gehe ins Volk (al. ich gehe ins Dorf $\rightarrow$ esp. Me voy al pueblo) o *ich habe mit meinem Bruder geschlafen (al. ich habe bei meinem Bruder geschlafen $\rightarrow$ esp. He dormido en casa de mi hermano). En el primer caso, y de acuerdo con lo observado en el proceso de enseñanza-aprendizaje del alemán como lengua extranjera, el origen del error puede estar relacionado con una incorrecta selección léxica como consecuencia de una lectura superficial del artículo lexicográfico. Si tomamos como referencia $\mathrm{P}-\mathrm{DBO}$ y $\mathrm{L}-\mathrm{DBO}$, el problema radica en que el usuario selecciona la primera acepción del sustantivo relativa al concepto de,pueblo‘ como nación. Siguiendo este razonamiento, si el usuario hubiera consultado Leo segu-

9. En diccionarios print esta información suele formar parte de la introducción, que generalmente no es consultada por los usuarios. En los DBO, sin embargo, el usuario no siempre recibe con un clic encima de las abreviaturas la información correspondiente, un aspecto, sin duda, mejorable. 
ramente no hubiera cometido este error de selección léxica, puesto que la primera acepción que recoge este diccionario es la de pueblo' como población pequeña.

En el segundo caso, el error es consecuencia de una incorrecta desambiguación sintáctica de los posibles equivalentes del verbo dormir. El verbo schlafen admite la preposición mit con el sentido de mantener relaciones sexuales con alguien y la preposición bei en el sentido de pernoctar o pasar la noche en casa de alguien. Como en el ejemplo anterior, los DBO consultados ofrecen de forma más o menos explícita la información necesaria para realizar una correcta desambiguación entre las formas schlafen mit y schlafen bei. En P-DBO la desambiguación se corresponde con las distintas acepciones del verbo schlafen. En la primera, con el significado de descansar, se ofrece el ejemplo mit jdm schlafen (dormir con alguien). En la segunda acepción, con el significado de pernoctar, se propone el siguiente ejemplo: de. Er / sie hat bei Juan übernachtet [o. geschlafen] (es. Ha dormido en casa de Juan). En Leo, por el contrario, la desambiguación schlafen mit/ schlafen bei únicamente tiene lugar en el foro mediante la intervención de un usuario; mientras que en L-DBO tan solo se hace referencia a la forma schlafen mit. Solo mediante la información complementaria que aporta OpenThesaurus, al cual se accede mediante un hipervínculo, se ofrece información relativa a dormir en el sentido de pernoctar, lo que lleva al usuario al equivalente übernachten en alemán, pero no a schlafen bei (dormir en casa de alguien).

Los ejemplos anteriores ponen de manifiesto que los errores en la producción o traducción en L2 como lengua meta derivados de la consulta del DB y, en este caso de DBO, no son consecuencia de una carencia informativa de la obra lexicográfica, sino de una mala interpretación de la misma (cfr. Porto Dapena 2002: 10). Con el fin de superar esta carencia y desde la perspectiva docente es fundamental introducir en el aula actividades que fomenten el desarrollo de las competencias lexicográficas, como por ejemplo, en el caso de la producción y traducción en L2, la capacidad de llevar a cabo una desambiguación a nivel morfológico, sintáctico, semántico, sintagmático-combinatorio y pragmático que derive en la selección adecuada del equivalente en la lengua meta y en su uso correcto en consonancia con una situación comunicativa concreta. Para ello, el primer paso es tomar conciencia de la información que pone a nuestra disposición el DBO, ya que, tal y como indica la encuesta DICONALE-DIC, los usuarios no explotan adecuadamente gran parte de la información y posibilidades que les ofrece la obra lexicográfica bilingüe (Meliss 2015b) ${ }^{10}$. Para concluir un proceso de consulta de forma satisfactoria en situaciones de codificación en L2, más allá de la búsqueda y selección del equivalente, el usuario debería plantearse una serie de preguntas en

10. Véase también la aportación de González y Meliss (2016) en la que se presentan los múltiples recursos online y su aplicación en la enseñanza de una lengua extranjera. 
torno a la forma, significado, combinatoria y uso de la unidad léxica que pretende seleccionar como posible equivalente del lema de la lengua de partida. Partiendo de estas formulaciones generales proponemos a continuación una serie de situaciones a modo de ejemplo que ayudarán a ilustrar la riqueza informativa de los DBO seleccionados, así como su valor como herramienta de aprendizaje.

Situación 1: un aprendiz de DaF de nivel inicial (A1) busca en el DBO el equivalente en alemán del sustantivo casa con el fin de producir un texto escrito.

\section{Entrada 5: primera acepción en el artículo lexicográfico de Haus en P-DBO} (fragmento)

\begin{tabular}{|c|c|c|}
\hline casa ['kasa] SUST $f$ & & e \\
\hline \multicolumn{3}{|l|}{ 1. casa (edificio): } \\
\hline * casa & Haus $n t$ & +6 \\
\hline * casa adosada & Reihenhaus $n t$ & +6 \\
\hline * casa de baños & Badehaus $n t$ & $\neq c$ \\
\hline * casa de campo & Landhaus $n t$ & $* 4$ \\
\hline * casa de citas & Bordell $n t$ & $\neq c$ \\
\hline * casa de comidas & Gaststätte $f$ & +6 \\
\hline * casa consistorial & Rathaus $n t$ & $\$ 6$ \\
\hline * casa de Dios & Gotteshaus $n t$ & +6 \\
\hline * casa de fieras & Tierhaus $n t$ & $\$ 4$ \\
\hline
\end{tabular}

Como se muestra en la entrada 5, el diccionario P-DBO proporciona al usuario en primer lugar el equivalente en la lengua meta: Haus ${ }^{11}$. Teniendo en cuenta el objetivo inicial del usuario, buscar el equivalente de casa en alemán, el proceso de consulta queda concluido. Sin embargo, si el usuario lo desea, el proceso de consulta puede continuar. En esta primera acepción [1. casa (edificio)] el diccionario propone una serie de sublemas en los que la palabra casa se combina con otras unidades léxicas. Se trata de una serie de compuestos endocéntricos frecuentes en español como casa adosada, casa de baños, etc., en los que el sustantivo casa funciona como núcleo semántico. Cada sublema cuenta con su equivalente en alemán como lengua meta, lo que puede inducir al usuario a reflexionar sobre la formación de los compuestos endocéntricos en alemán. Los compuestos en español presentan dos realizaciones morfosintácticas diferentes: $\mathrm{S}+\mathrm{Adj}$ (casa adosada) y $\mathrm{S}+\mathrm{de}+\mathrm{S}$ (casa de baños). Se trata, por lo tanto, de compuestos sintagmáticos. Los equivalentes en alemán, por el contrario, adoptan en general la forma de compuestos ortográficos, en los que el núcleo semántico, a diferencia del

11. Cabe destacar que el usuario no recibe aquí información sobre el paradigma de flexión, por lo que debe conocer los pasos a seguir para lograr saber, por ejemplo, el plural del sustantivo (pinchar en Haus y llegar a la parte del diccionario alemán-español o buscar un cuadro de flexión mediante un link externo). 
español, va en última posición, detrás del elemento modificador (casa adosada = Reihenhaus, casa de baños = Badehaus). Esta observación lleva al usuario a formular una hipótesis inicial sobre la formación de compuestos en alemán ${ }^{12}$, que, posteriormente, podrá contrastar y verificar en manuales de gramática o con la ayuda del docente. Lo importante en este caso es el proceso inductivo que pone en marcha el aprendiz a partir de una consulta en el DBO, en el que, más allá de la información explícita (ortografía, género gramatical, etc.), le invita a reflexionar sobre un aspecto concreto del funcionamiento de la unidad léxica en el sistema lingüístico de la lengua meta.

Situación 2: un aprendiz de DaF de nivel inicial (A1) busca en el DB el equivalente en alemán del adjetivo rico con el fin de producir un texto oral. Su compañero de piso alemán ha decidido cocinar y le gustaría poder alabar sus dotes culinarias. Como se observa en la entrada 6, L-DBO ofrece la siguiente información:

\section{Entrada 6: artículo lexicográfico de rico en L-DBO}

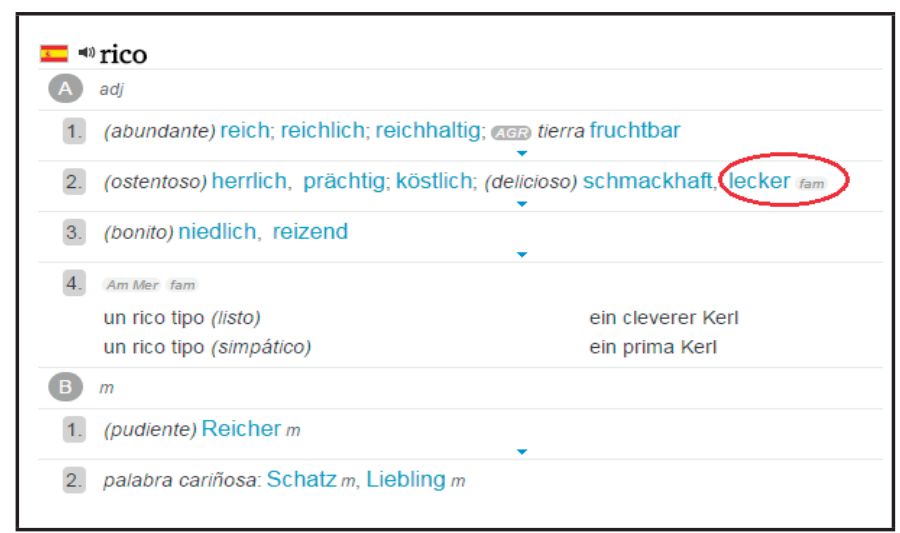

El usuario identifica fácilmente la primera acepción como la más adecuada para sus fines comunicativos ya que el diccionario propone una desambiguación semántica estructurada de la lengua de partida y, dentro de esta, la subacepción número 2 con el sentido de 'delicioso'. El usuario solo tendrá que elegir entre dos posibles equivalentes: schmackhaft y lecker. El diccionario proporciona una marca de restricción diafásica que puede ayudar en la selección del equivalente léxico. Junto al adjetivo lecker aparece la abreviatura "fam." que adscribe el uso de este adjetivo al registro coloquial. Teniendo en cuenta el contexto comunicativo concreto del usuario, lecker se perfila como la mejor opción. Puesto que el objetivo del

12. Como se observa en la entrada 5, no siempre se trata en alemán de compuestos con el elemento base Haus. Este hecho pone de manifiesto la asimetría entre ambas lenguas, un aspecto sobre el que el usuario también podría reflexionar. 
usuario es la producción de un texto oral, quizás necesite también información que le ayude a reproducir oralmente la unidad léxica. Para ello, si pincha sobre el adjetivo lecker, el DBO le dirige al artículo lexicográfico en el que el alemán constituye la lengua de partida y, donde además de la marca de sílaba tónica ('lecker), pone a su disposición un archivo de audio en el que un hablante nativo pronuncia la palabra ( $c f r$. entrada 7). Si el usuario posee una buena comprensión auditiva se percatará además de una discrepancia entre la secuencia grafemática de <lecker> y la realización fónica (la segunda sílaba se realiza en el fichero audio con una "R" vocálica y no consonántica). Si el usuario sigue interesándose por esta cuestión, encontrará la transcripción fónica en IPA en el P-DBOः lecker[ [kk] ], cuya correcta interpretación depende de los conocimientos fonéticos del usuario.

\section{Entrada 7: artículo lexicográfico de lecker en L-DBO}

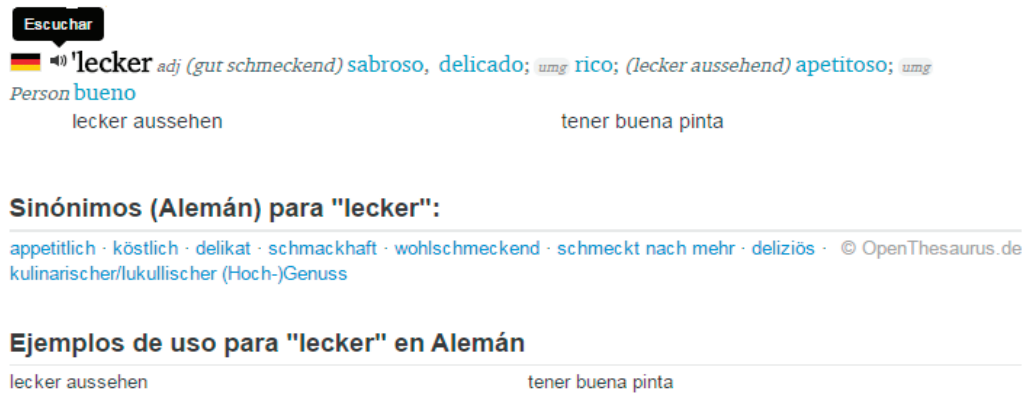

Además del archivo de audio, el diccionario ofrece al usuario un ejemplo recurrente de uso en combinación con el verbo aussehen y una amplia compilación de sinónimos del adjetivo lecker a partir de la cual puede seguir profundizando su búsqueda inicial hasta, por ejemplo, elaborar un paradigma semántico en torno al concepto de 'rico' en la $\mathrm{L} 2^{13}$.

13. Sobre el tratamiento del lenguaje hablado en los diccionarios de aprendizaje del alemán como lengua extranjera véase Meliss (2016a). 
Situación 3: un aprendiz de DaF de nivel intermedio (B1) busca en el DBO el equivalente en alemán del verbo ordenar con el fin de traducir a la lengua meta la oración el sargento ordenó el ataque a la isla.

\section{Entrada 8: artículo lexicográfico de ordenar en Leo (fragmento)}

\begin{tabular}{|c|c|}
\hline \multicolumn{2}{|c|}{ Verben } \\
\hline 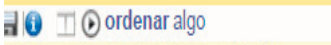 & I(1) etw. Atk. ordnen | ordnete, geordnet| \\
\hline 30 I0 Ordenar algo - piso, etc. & 7. (1) etw. Akk. aufräumen | räunte auf, aufgeräunt| - Wohnung etc. \\
\hline 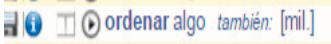 & (1) etw. AblD Defehlen | befahl, befohlen | \\
\hline
\end{tabular}

En el artículo lexicográfico del verbo ordenar propuesto en Leo (cfr. entrada 8) el usuario encuentra información fundamental para proceder a una desambiguación léxica satisfactoria en la lengua de partida que le permite seleccionar adecuadamente el equivalente en alemán. En la tercera acepción, el diccionario incorpora la marca diafásica "mil." relativa al contexto militar. Una vez seleccionado el equivalente en la lengua meta, befehlen, el usuario necesita información para su correcto uso. Leo explicita parte de la estructura argumental del verbo mediante información morfosintáctica: etw. Akk. befeblen, además de las formas irregulares del Präteritum (befabl) y del Partizip II (befoblen). Esta información cubriría las necesidades del usuario acorde con el objetivo principal de su consulta; sin embargo, como en los ejemplos anteriores, la consulta puede continuar. Tomando conciencia de que el verbo befehlen posee un paradigma de flexión fuerte, el usuario podría interesarse por la conjugación completa del verbo. Leo pone a su disposición esta información a través de un hipervínculo que le dirige a la tabla de conjugación. Como apuntamos arriba, la información proporcionada por Leo sobre la estructura argumental del verbo befeblen es suficiente para satisfacer las necesidades del usuario. En este caso el complemento directo se realiza mediante una frase nominal, por lo que la oración en la lengua meta presenta una estructura semejante a la de la lengua de partida: de. der Unteroffizier befabl den Angriff auf die Insel. A partir de este ejemplo el usuario podría preguntarse qué representación sintáctica tendría en la lengua meta una oración del tipo el sargento ordenó que atacaran la isla, en la que el complemento directo en español adopta una forma oracional. En este contexto la información proporcionada en Leo sería insuficiente $e^{14}$.

Situación 4: un aprendiz de DaF de nivel inicial (A2) busca en el DB el equivalente en alemán de la unidad plurilexemática dar un paseo con el fin de producir

14. El usuario podría consultar para este caso concreto otros recursos lexicográficos online como Linguee o Bab.la. 
un texto escrito. El uso del $\mathrm{DBO}$ en este ejemplo requiere ya de una mínima competencia lexicográfica. El usuario debe saber que para buscar construcciones verbonominales hay que partir de la entrada referente al sustantivo, en este caso paseo.

Entrada 9: artículo lexicográfico de paseo en L-DBO (fragmento)

\begin{tabular}{|c|c|c|}
\hline \multicolumn{3}{|c|}{ 도 " paseom } \\
\hline \multirow[t]{5}{*}{1.} & a pie: Spaziergang $m$; con & a caballo: Spazierritt $m$ \\
\hline & paseo en coche & Kutschfahrt $f$ \\
\hline & paseo en trineo & Schlittenpartie $f$ \\
\hline & dar un paseo & einen Spaziergang machen \\
\hline & dar un paseo por las calles & durch die Straßen schlendern \\
\hline
\end{tabular}

Como se muestra en la entrada 9, el usuario puede identificar fácilmente el equivalente ya que en la primera acepción del sustantivo se propone el ejemplo dar un paseo con su equivalente en la lengua meta: einen Spaziergang machen. Lo interesante en este caso es el proceso de reflexión que puede provocar en el usuario el contraste entre ambas lenguas en relación a esta expresión. En la construcción española, el sustantivo paseo se combina con el verbo dar; mientras que en alemán el sustantivo Spaziergang se combina con el verbo machen (hacer) y no con el verbo geben (dar). El usuario podría preguntarse por qué en español damos un paseo y en alemán se «hace un paseo» e intentar averiguar si se trata de un caso aislado o si, por el contrario, puede establecerse una tendencia divergente en la selección verbal de este tipo de construcciones en alemán y español. De esta forma, el usuario podría interesarse por los equivalentes de construcciones como dar un beso, dar un abrazo o darse una ducha con el fin de comprobar si, al igual que para dar un paseo, el alemán seleccionar el verbo machen $^{15}$.

\section{Conclusiones y desiderata}

De acuerdo con las tres competencias lexicográficas básicas presentadas y partiendo de los ejemplos anteriormente expuestos podemos resumir que el usuario necesita identificar adecuadamente la información lingüística y extralingüística pertinente en cuanto a la forma, el contenido y su uso tanto para la situación de recepción y producción en L2 como para la traducción de/a L2. La identificación explícita e implícita de esta información junto con el aprovechamiento de las ca-

15. Dar un beso (al. einen Kuss geben), dar un abrazo (al. eine Umarmung geben) y darse una ducha (al. eine Dusche nebmen). 
racterísticas específicas de un diccionario en formato online y el conocimiento de las diferentes formas de acceso a la información deben formar parte de la competencia lexicográfica de cualquier aprendiz de una lengua extranjera. El desarrollo de estas competencias enlaza directamente con la importancia del diccionario bilingüe más allá de su uso tradicional como proveedor de equivalentes léxicos y con la necesidad de introducir una didáctica lexicográfica en el aula de lengua extranjera. De la misma manera, se debe promover la elaboración de diccionarios adaptados a las nuevas necesidades de los usuarios en el contexto de una sociedad digitalizada (cfr. Meliss / Sánchez. Hernández 2015) en base a los resultados que aportan los estudios empíricos en la investigación sobre el uso de diccionarios (cfr. Koplenig / Müller-Spitzer 2014). En el ámbito universitario y a pesar de la introducción de la didáctica lexicográfica en los currículos de la enseñanza secundaria los estudiantes tienen dificultades para interpretar y explotar adecuadamente la información lexicográfica.

Teniendo en cuenta lo anterior formulamos los siguientes desiderata, por un lado en cuanto a algunas de las necesidades para la mejora de la didáctica lexicográfica y, por otro, en relación a algunas exigencias para una nueva generación de diccionarios pedagógicos bilingües en formato online:

+ desarrollo de una didáctica lexicográfica actual que contemple los nuevos formatos,

- inclusión de una didáctica lexicográfica en todas las asignaturas de lengua, tanto de lenguas maternas como extranjeras,

- formación del profesorado de lenguas en una didáctica lexicográfica actual $^{16}$,

- elaboración de diccionarios pedagógicos que contemplen con mayor interés los diferentes usuarios y situaciones de uso $^{17}$,

- presentación de la información lexicográfica con un grado más elevado de visualización y

+ promoción de un manejo más sencillo e intuitivo de la obra lexicográfica.

16. Con el fin de conocer la formación lexicográfica del profesorado de español y alemán como lenguas extranjeras y promover un mayor interés por el uso del diccionario en el aula se ha elaborado una encuesta en línea: DICONALE-DIC-DOC cuyos resultados se publicarán en Meliss/Egido Vicente/Fernández Méndez (2018).

17. En este sentido se están haciendo actualmente esfuerzos para diferentes lenguas. Entre otros ejemplos citamos eldit para el alemán-italiano y el proyecto DICONALE (cfr. Egido et alii 2015, González y Meliss 2015, Meliss 2015a, 2016c, Meliss y Sánchez 2015). 


\section{Referencias bibliográficas}

Artículos y monografías:

Abel, A. y Lemnitzer, L. (eds.) (2014). «Vernetzungsstrategien, Zugriffsstrukturen und automatisch ermittelte Angaben in Internetwörterbüchern». Opal, vol. 2, Mannheim: IDS. [<http://pub.idsmannheim.de/laufend/opal/pdf/opal2014-2.pdf > ] (07/04/2017)

Egido Vicente, M. (2011). «El papel del diccionario bilingüe y la didáctica lexicográfica en la enseñanza de ELE a germanoparlantes». ZRomSD, Stuttgart, Ibidem, vol. 5,2: 43-60.

Egido Vicente, M., Fernández Méndez, M. y Franco Barros, M. (2015). «A new pedagogical dictionary for DAF and ELE with an onomasiological focus». En: J. Paulo Silvestre y A. Villalva. (eds.). Planning non-existent dictionaries. Lisboa / Aveiro. Centro de Lingüística da Universidades de Lisboa / Universidades de Aveiro: 143-150.

Engelberg, S. y Lemnitzer, L. (2001, $\left.{ }^{4} 2009\right)$. Lexikographie und Wörterbuchbenutzung. Tübingen: Stauffenburg.

Engelberg, S. y Müller-Spitzer, C. (2013). «Dictionary Portals». En: R. H. Gouws et alii (eds.). Dictionaries. An international encyclopedia of lexicography. Supplementary volume: Recent developments with Focus on Electronic and Computational Lexicography. Berlin/New York. de Gruyter: 1023-1034.

Fernández Méndez, M. (2015): «Argumentstrukturmuster und valenzgrammatisch orientierte Information im deutsch-spanischen Kontrast. Exemplarische Analyse der Lexikalisierungsmöglichkeiten des Konzepts ENTFÜHREN». En St. Engelberg et alii (eds.). Argumentstruktur zwischen Valenz und Konstruktion. Tübingen. Narr: 353-364.

Fernández Méndez, M. et alii (2016): «Uso de diccionarios de aprendizaje: Análisis de una encuesta desde una perspectiva contrastiva sobre el uso de los diccionarios bilingües alemán-español entre aprendices de ELE y DaF». En F. Robles et alii. (eds.). Sprachdidaktik Spanisch - Deutsch. Forschungen an der Schnittstelle von Linguistik und Fremdsprachendidaktik.Tübingen: Narr Francke Attempto: 73-92.

Flinz, C. (2014). «Wörterbuchbenutzung: Ergebnisse einer Umfrage bei italienischen DaF-Lernern ». En A. Abel et alii. (eds.) (2014). Proceedings of the XVI Euralex international Congress: The User in Focus. 15-19 July 2014. Bolzano / Bozen. Institute for Specialised Communication and Multilingualism: 213-224. [<http://www.euralex.org/proceedings-toc/ euralex_2014/>] (07/04/2017). 
Fuentes Morán, M. T. (1997). Gramática en la lexicografía bilingüe. Morfología y sintaxis en diccionarios español-alemán desde el punto de vista del germanohablante. Tübingen: Niemeyer.

Fuentes Morán, M. T. (2015). «Representación del anisomorfismo en diccionarios bilingües alemán-español». En M. Meliss y B. Pöll (eds.). Aktuelle Perspektiven der kontrastiven Sprachwissenschaft: Deutsch-SpanischPortugiesisch. Zwischen Tradition und Innovation. Tübingen. Narr: 285-297.

Fuertes-Olivera, P. A. y Bergenholtz, H. (eds.) (2011). elexicography. The Internet. Digital Initiatives and Lexicography. London / New York: Continuum. González Ribao, V+y Meliss, M. (2015)• «Theoretische und methodologische Grundlagen eines onomasiologisch-konzeptuell orientierten Produktionswörterbuches für den zweisprachigen Lernerkontext: deutschSpanisch». En: C. Calañas Continente y F. Robles i Sabater. (eds.). Die Wörterbücher des Deutschen: Entwicklungen und neue Perspektiven. Frankfurt a. M. P. Lang: 109-136.

González Ribao, V+y Meliss, M. (2016). «Wortprofile, Kookkurrenzen und Konkordanzen in Forschung und Lehre im hispanophonen DaF-Umfeld: Bestandsaufnahme und -beschreibung für kontastive Aufgabenstellungen». En:F.RoblesiSabater etalii(eds.).SprachdidaktikSpanisch-Deutsch.Forschungen an der Schnittstelle von Linguistik und Fremdsprachendidaktik. Tübingen: Narr/Francke/Attempto (Reihe: Romanistische Fremdsprachenforschung und Unterrichtsentwicklung, Bd. 6): 93-112.

Gouws, R. H. et alii. (eds.). (2013). Wörterbücher / Dictionaries / Dictionnaires. Supplementary Volume Dictionaries. An International Encyclopedia of Lexicography. HSK 5/4. Berlin: de Gruyter Mouton.

Hausmann, F. J. (1991). «Die zweisprachige Lexikographie Spanisch-Deutsch, Deutsch-Spanisch». En: F. J. Hausmann et alii. (eds.). Dictionaries. An international Encyclopedia of Lexicography. HSK 5.3. Berlin. de Gruyter: $2987-2991$.

Hausmann, F. J. y Werner, R. (1991). «Spezifische Bauteile und Strukturen zweisprachiger Wörterbücher: eine Übersicht». En* F+ J. Hausmann et alii. (eds.). Dictionaries. An international Encyclopedia of Lexicography. HSK 5.3. Berlin. de Gruyter. 2729- 2770.

Hass U. y Sснмiтz U. (2010). «Lexikographie im Internet». Lexicographica, Berlín, de Gruyter, vol. 26-1-18.

HeId, U. (2013). «The impact of computational lexicography». En* R. H. Gouws et alii. (eds.). Wörterbücher / Dictionaries / Dictionnaires. Supplementary 
Volume Dictionaries. An International Encyclopedia of Lexicography. HSK 5/4, Berlin. de Gruyter Mouton: 24-30.

Kemmer, K. (2010). «Onlinewörterbücher in der Wörterbuchkritik. Ein Evaluationsraster mit 39 Beurteilungskriterien». OPAL, vol. 2, Mannheim: IDS.[<http://pub.ids-mannheim.de/laufend/opal/pdf/opal2010-2.pdf > ] $(07 / 04 / 2017)$

Klosa, A. (ed.). (2008). «Lexikographische Portale im Internet». OPALSonderheft, vol. 1, Mannheim: IDS.: [http://pub.ids-mannheim.de/laufend/ opal/pdf/opal2008-1.pdf] (07/04/2017)

Klosa, A. y Müller-Spitzer, C. (ed.) (2016): Internetlexikografie: ein Kompendium. Berlin / Boston, de Gruyter.

Koplenig, A. y Müller-Spitzer, C. (2014). «General issues of online dictionary use». En: C. Müller-Spitzer. (ed.). Using online dictionaries. Berlin / New York. de Gruyter: 127- 142.

Mann, M. (ed.). (2014). Digitale Lexikographie. Ein- und mehrsprachige elektronische Wörterbücher mit Deutsch: aktuelle Entwicklungen und Analysen (Reihe: Germanistische Linguistik, vol. 223-224). Hildesheim: Olms.

Melchior,L.(2012). «Halbkollaborativität und Online-Lexikographie. Ansätze und Überlegungen zu Wörterbuchredaktion und Wörterbuchforschung am Beispiel LEO Deutsch-Italienisch». Lexicographica, Berlin, de Gruyter, vol. 28. 337-372.

Meliss, M. (2013). «Das zweisprachige Wörterbuch im bilateralen deutschspanischen Kontext. Alte und neue Wege». En: M. J. Domínguez Vázquez (ed.). Trends in der deutsch-spanischen Lexikographie. Frankfurt a. M. Peter Lang: 61-87.

Meliss, M. (2015a). «Propuestas para un diccionario conceptual bilingüe para Ele y DaF: ¿Qué aportan los diccionarios de aprendizaje para situaciones de expresión escrita en lengua extranjera?». En: M. J. Domínguez VÁzquez et alii. (eds.). Lexicografía de las lenguas románicas. Aproximaciones a la lexicografía moderna y contrastiva. Berlin / München / Boston. de Gruyter: 271-296.

Meliss, M. (2015b). «Was suchen und finden Lerner des Deutschen als Fremdsprache in aktuellen Wörterbüchern? Auswertung einer Umfrage und Anforderungen an eine aktuelle DaF-Lernerlexikographie». En: Th. Roelcke, Th. (ed.). Wörterbücher für Deutsch als Fremdsprache - Probleme und Perspektiven. Themenreihe. InfoDaF 4/42. München. Iudicium: 401-432. [<http://www.daf. de/downloads/InfoDaF_2015_Heft_4.pdf >] (07/04/2017)

Meliss, M. (2016a). «Gesprochene Sprache in DaF-Lernerwörterbüchern». En: B. Handwerker et alii (eds.). Gesprochene Fremdsprache 
Deutsch. Baltmannsweiler: Schneider Verlag Hohengehren (Reihe: Perspektiven Deutsch als Fremdsprache, vol. 32): 179-199.

Meliss, M. (2016b). «Wie viele und welche bilingualen Online-Wörterbücher brauchen wir für den DaF-Bereich? Erstellung eines Kriterienrasters und erste Bestandsaufnahme aus der Sicht eines hispanophonen Lernerkontextes». En: J. Cuartero Otal et alii (eds.). Querschnitt durch die deutsche Sprache aus spanischer Sicht. Perspektiven der kontrastiven Linguistik. Berlin: Frank \& Timme: 187-210.

Meliss, M. (2016c). «Lexikalische Vielfalt und Varianz aus kontrastiver Perspektive: Überlegungen zu einem Produktionswörterbuch aus der Sicht des Deutschen und Spanischen». En: L. Borex y A. Rapp (eds.). Varianz und Vielfalt interdisziplinär. Wörter und Strukturen. Opal, vol. 2, Mannheim: IDS. [<http //pub.ids-mannheim.de/laufend/opal/pdf/opal2016-2.pdf $>$ ] (07/04/2017)

Meliss, M., Egido Vicente, M. y Fernández Méndez, M. (2018 e.p.). « Welche Information versteckt sich in Online-(Lerner)wörterbüchern? Plädoyer für die Entwicklung einer digital-lexikografischen Kompetenz im Fremdsprachenunterricht ». En: A. Klosa et alii (eds.). Lexicographica 2018 (parte temática: Internetlexikographie und Sprachvermittlung).

Meliss, M. y Sánchez Hernández, P. (2015). «Nuevos retos para la lexicografía pedagógica, bilingüe alemán-español». RILE, vol. 4: 123-150. [<http://revistes.publicacionsurv.cat/index+php/rile/issue/view/60/ show Toc > ] (07/04/2017).

Meyer, Ch. y Gurevych, I. (2014). «Methoden bei kollaborativen Wörterbüchern». Lexicographica, Berlin, de Gruyter, vol. 30: 87-212.

Meyer, M. y Wiegand, H. E. (2000). «Gemischt-semintegrierte Mikrostrukturen für deutsch-spanische Printwörterbücher». En H. E. Wiegand (ed.). Studien zur zweisprachigen Lexikographie mit Deutsch. Hildesheim. Olms: 87-171.

Model, B. (2010). Syntagmatik im zweisprachigen Wörterbuch. Berlin: De Gruyter.

Nied-Curcio, M. (2014). «Die Benutzung von Smartphones im Fremdsprachenerwerb und -unterricht». En A. Abel et alii. (eds.) (2014). Proceedings of the XVI Euralex international Congress: The User in Focus. 15 19 July 2014. Bolzano / Bozen. Institute for Specialised Communication and Multilingualism*263-280. [<http //www.euralex.org/proceedings-toc/ euralex_2014/>] (07/04/2017). 
Porto Dapena, J. A. (2002). Manual de técnica lexicográfica. Madrid: Arco Libros.

Schmitz, U. (2013). «Monolingual and bilingual electronic dictionaries on the Internet». En R. H. Gouws et alii. (eds.). Wörterbücher / Dictionaries / Dictionnaires. Supplementary Volume Dictionaries. An InternationalEncyclopedia of Lexicography. HSK 5/4. Berlin. de Gruyter Mouton: 1013-1022.

Storrer, A. (2010). «Deutsche Internet-Wörterbücher: Ein Überblick». Lexicographica. Berlin / New York, de Gruyter, vol. 26: 154-164.

TARP, S. (2013). «New developments in learners' dictionaries III: Bilingual learners' dictionaries». En R. H. Gouws et alii. (eds.). Wörterbücher / Dictionaries / Dictionnaires. Supplementary Volume Dictionaries. An International Encyclopedia of Lexicography. HSK 5/4. Berlin. de Gruyter Mouton: 425-430.

\section{Recursos lexicográficos online}

BAB.LA: http://bab.la/ (07/04/2017)

Diconale: https://www.usc.es/es/proxectos/diconale/ (07/04/2017)

Dict: http://www.dict.cc/ (07/04/2017)

ELDIT: http://eldit.eurac.edu/ (07/04/2017)

L-DBO: Langenscheidt diccionario bilingüe online: http://de.langenscheidt. com/ (07/04/2017)

LEO: https://dict.leo.org/ende/index_de.html (07/04/2017)

LinguATOols: http://www.linguatools.de/ (07/04/2017)

LiNGUEEः http://www.linguee.es/ (07/04/2017)

Open Thesaurus: https://www.openthesaurus.de/ (07/04/2017)

P-DBO: Pons diccionario bilingüe online: http://de.pons.com/ (07/04/2017) 